\title{
Image Segmentation and Retrievals based on Finite Doubly Truncated Bivariate Gaussian Mixture Model and K-Means
}

\author{
Rajkumar G.V.S. \\ Department of IT, \\ GITAM University \\ Visakhapatnam-45
}

\author{
Srinivasa Rao K. \\ Department of Statistics \\ Andhra University \\ Visakhapatnam-03
}

\author{
Srinivasa Rao P. \\ Department of CS\&SE \\ Andhra University \\ Visakhapatnam-03
}

\begin{abstract}
A new Image Segmentation method based on Finite Doubly Truncated Bivariate Gaussian Mixture Model is proposed in this paper. The Truncated Bivariate Gaussian Distribution includes several of the skewed and asymmetric distributions as particular cases with finite range. This distribution also includes the Gaussian distribution as a limiting case. We use Expectation maximization (EM) algorithm to estimate the model parameters of the image data and the number of mixture components is estimated by using $K$-means Clustering algorithm. The $K$-means clustering algorithm is also utilized for developing the initial estimates of the EMalgorithm. The segmentation is carried out by clustering of feature vector into appropriate component according to the Maximum Likelihood Estimation criteria. The advantage of our method lies its efficiency on initialization of the model parameters and segmenting the images in a totally unsupervised manner. The performance of the proposed algorithm is studied by computing the segmentation performance measures like, PRI, GCE and VOI. The ability of this method for image retrieval is demonstrated by computing the image quality metrics for six images namely OSTRICH, POT, TOWER, BEARS, DEER and BIRD. The experimental results show that this method outperforms the existing model based image segmentation methods.
\end{abstract}

Keywords: Image Segmentation, Truncated Bivariate Gaussian Mixture Distribution, Image Quality Metrics, K-means algorithm, EM-algorithm.

\section{INTRODUCTION}

Image analysis is an important area of research in image processing and image retrievals. In image analysis the basic consideration is extracting useful information from the images through features. Image analysis involves a low level and a high level processing. In low level analysis, the representation of an image is transformed from a numerical array of pixel intensities to symbolic set of image primitives (edges and regions). In high level analysis, object labels (or interpretations) are assigned to these primitives, there by providing a semantic description of the image (Modestino J.W. and Zhang J. (1992)). Image analysis techniques can be classified into two major groups: 1) Statistical, which uses probability distribution functions of pixels and regions to characterize the image (Dubes R.C. and Jain A.K. (1989)), and 2) Structural, which analyzes the image in terms of organization and relationship of pixels and regions by the specified relations (Udapa J.K. et al (1996)). Image segmentation is defined as the process of dividing the image into different regions, such that each region is homogenous. For intensity image segmentation there exists three popular approaches namely, i) histogram analysis technique ii) region growing and iii) edge detection. A more comprehensive discussion on image segmentation, were presented by Pal S.K. and Pal N.R. (1993), Jahne (1995), Cheng et al (2001), Ye Hou et al (2009), Un Tang (2010), Nor Hazlyna et al (2010), Laurent Najman (2011), Juyong Zhang (2010),. There does not exist a unique algorithm that works for all applications.

In low level image analysis, the segmentation gained lot of importance due to its more accuracy in extracting information from the image. Hence, segmentation becomes the first step of low level image analysis. Segmentation is a process of dividing whole image into non overlapping regions such that each region is homogeneous. Several image segmentation techniques have been developed and utilized for the image analysis depending upon situation under consideration.

Recently, much emphasis is given to utilize model based image segmentation by Srinivas Y. et al (2010), Zhen Wang et al (2010), Feng Zhu et al (2010), Kokkinos I. et al (2009), Zhenh a Zhang et al (2009), Raut S. (2009). In model based image segmentation the whole image is characterized through finite Gaussian mixture model (GMM) (Lie T. et al (1993), Yamazaki T. et al. (1998), Zhang Z.H. et al (2003)). In finite Gaussian mixture models, each image region is characterized by a Gaussian distribution. This finite Gaussian mixture model serves well only when the ran ge of feature vector is infinite. But in many image regions the pixel intensities will have a finite range. Taking this into consideration Srinivas Y. and Srinivas Rao K. (2007) developed an unsupervised learning algorithm using finite truncated Gaussian mixture model assuming that the pixel intensities in each region follows a univariate truncated Gaussian distribution. This model is suitable for gray images. But in colour images the pixel intensities can be well characterized by vector of features in colour space.

Several colour spaces are proposed for different contexts of image processing. Some of the well known colour spaces are RGB, HSV, YIQ, and CIE spaces. Among these the RGB space is quite commonly used because of its simplicity in implementation. A better colour space than the RGB space in representing the colours of human perception is the HSV space, in which the colour information is represented by Hue and Saturation values. Thus the human perception of image can be characterized through a bivariate random variable consisting of Hue and Saturation which can be measured through using generic structure of a colour appearance model (San gwine et al (1998)).

Hence, in this paper, an image segmentation algorithm with the assumption that the feature vector of the colour image is represented by a two dimensional variable namely, Hue angle and 
Saturation is developed and analyzed. It is assumed that the whole image is characterized by finite doubly truncated bivariate Gaussian mixture distribution and the numbers of components in the mixture are identified through K-means algorithm (Rose H. Turi (2001)) along with the histogram of the pixel intensities. Deriving the updated equations of the EM - algorithm the model parameters are estimated. The segmentation is carried through maximizing the component likelihood function.

The performance of the developed segmentation algorithm is studied by deriving performance measures namely, Probabilistic Rand Index (PRI), Global Consistency Error (GCE) and the Variation of Information (VOI) and comparing them with existing model based segmentation methods with six images namely, OSTRICH, POT, TOWER, BEARS, DEER and BIRD. The segmentation algorithm is used to reconstruct the image and its performance is studied through image quality metrics like, Maximum distance, Image fidelity, Mean square error, Signal to noise ratio and Image quality index and comparing it with earlier algorithms.

\section{TRUNCATED BIVARIATE GAUSSIAN MIXTURE MODEL}

In a low level image analysis, the entire image is considered as a union of several image regions. The CIE spaces provide an approximately uniform chromaticity scale, which allows the use of Euclidean distance in expressing the colour difference of human perception, and thus is especially efficient in the measurement of small colour difference (Tseng D.C. et al (1992)). Among all those colour spaces the bivariate features Hue and Saturation of the image derived from CIE- $\mathrm{L}^{*} \mathrm{U}^{*} \mathrm{~V}^{*}$ measures formulate a bivariate feature vector.

Let $\mathrm{W}=(\mathrm{X}, \mathrm{Y})$ is a bivariate random variable representing feature vector of pixel of a image region. Since these two variables are highly influenced by various random factors like vision, lighting, moisture, environmental conditions, etc, this feature vector can be viewed as a bivariate random vector. To model the bivariate features of the image, it is customary to assume that the feature vector of the image follows a bivariate Normal (Gaussian) distribution. The probability density function of the bivariate normal distribution is,

$$
\begin{aligned}
f(x, y)=\frac{1}{2 \pi \sqrt{1-\rho^{2}} \sigma_{1} \sigma_{2}} \exp \left\{\frac{-1}{2\left(1-\rho^{2}\right)}\left[\left(\frac{x-\mu_{1}}{\sigma_{1}}\right)^{2}-2 \rho\left(\frac{x-\mu_{1}}{\sigma_{1}}\right)\left(\frac{y-\mu_{2}}{\sigma_{2}}\right)+\left(\frac{y-\mu_{2}}{\sigma_{2}}\right)^{2}\right]\right\} \\
-\infty<x<+\infty ;-\infty<y<+\infty, \\
\\
\sigma_{1}>0 ; \sigma_{2}>0 ;-1<\rho<1, \\
-\infty<\mu_{1}<+\infty ;-\infty<\mu_{2}<+\infty
\end{aligned}
$$

However, in many practical situations the elements of the feature vector of the image region lie between two finite values and in some image regions the distribution may be asymmetric and skewed. Neglecting the reality of finite range leads to serious falsification of the model estimation. To incorporate the phenomenon of finite range of the vector, the probability distribution associated with the feature vector is modified with truncation at tail ends. The probability density function of the doubly truncated Gaussian distribution is given by,

$$
g(x, y ; \theta)=\frac{f(x, y)}{\int_{b_{1}} b_{2} \int_{2} f(x, y) d x d y}, \mathrm{~b}_{1}<x<\mathrm{a}_{1} ; \mathrm{b}_{2}<y<\mathrm{a}_{2}
$$

where, $\left(b_{1}, a_{1}\right)$ are the truncation points of the Hue and $\left(b_{2}, a_{2}\right)$ are the truncation points of the Saturation. $f(x, y)$ is the probability density function of the bivariate Normal distribution given in equation(1).

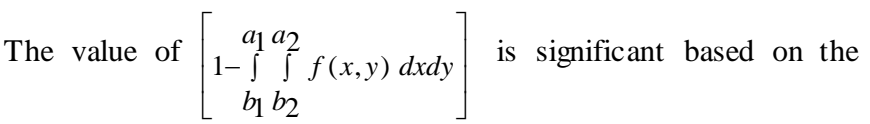
values of the parameters. This distribution includes the skewed, asymmetric and finite range bivariate distributions as particular cases for limiting and specific values of the parameters. This model also includes bivariate Gaussian distribution as a limiting case. The effect of truncation in bivariate Gaussian distribution has been discussed by several researchers (Norman L. Johnson, Samuel Kotz and Balakrishnan (1994)).

Following the heuristic arguments given by Bengt Muthen (1990), the mean value of ' $\mathrm{X}$ ' (hue) is

$\mathrm{E}(\mathrm{X})=\mu_{1}+\sigma_{1} \mathrm{~A}$

where,

$$
\begin{aligned}
\mathrm{A}=[ & -\phi\left(\frac{a_{1}-\mu_{1}}{\sigma_{1}}\right)\left[\Phi\left[\left(\left(\frac{a_{2}-\mu_{2}}{\sigma_{2}}\right)-\rho\left(\frac{a_{1}-\mu_{1}}{\sigma_{1}}\right)\right) c\right]-\Phi\left[\left(\left(\frac{b_{2}-\mu_{2}}{\sigma_{2}}\right)-\rho\left(\frac{a_{1}-\mu_{1}}{\sigma_{1}}\right)\right) c\right]\right] \\
& +\phi\left(\frac{b_{1}-\mu_{1}}{\sigma_{1}}\right)\left[\Phi\left[\left(\left(\left(\frac{a_{2}-\mu_{2}}{\sigma_{2}}\right)-\rho\left(\frac{b_{1}-\mu_{1}}{\sigma_{1}}\right)\right) c\right]-\Phi\left[\left(\left(\frac{b_{1}-\mu_{1}}{\sigma_{1}}\right)-\rho\left(\frac{b_{1}-\mu_{1}}{\sigma_{1}}\right)\right) c\right]\right]\right. \\
& -\rho \phi\left(\frac{a_{2}-\mu_{2}}{\sigma_{2}}\right)\left[\Phi\left[\left(\left(\left(\frac{a_{1}-\mu_{1}}{\sigma_{1}}\right)-\rho\left(\frac{a_{2}-\mu_{2}}{\sigma_{2}}\right)\right) c\right]-\Phi\left[\left(\left(\frac{b_{1}-\mu_{1}}{\sigma_{1}}\right)-\rho\left(\frac{a_{2}-\mu_{2}}{\sigma_{2}}\right)\right) c\right]\right]\right. \\
& +\rho \phi\left(\frac{b_{2}-\mu_{2}}{\sigma_{2}}\right)\left[\Phi\left[\left(\left(\left(\frac{a_{1}-\mu_{1}}{\sigma_{1}}\right)-\rho\left(\frac{b_{2}-\mu_{2}}{\sigma_{2}}\right)\right) c\right]-\Phi\left[\left(\left(\frac{b_{1}-\mu_{1}}{\sigma_{1}}\right)-\rho\left(\frac{b_{2}-\mu_{2}}{\sigma_{2}}\right)\right) c\right]\right]\right]
\end{aligned}
$$

and $c=\left(1-\rho^{2}\right)^{-1 / 2}, \phi, \Phi$ are the ordinate and area of a standard Normal distribution.

Similarly the mean value of ' $\mathrm{Y}$ '(saturation) is

$\mathrm{E}(\mathrm{Y})=\mu_{2}+\sigma_{2} \mathrm{~B}$

where,

$$
\begin{aligned}
\mathrm{B}=[ & -\phi\left(\frac{a_{2}-\mu_{2}}{\sigma_{2}}\right)\left[\Phi\left[\left(\left(\frac{a_{1}-\mu_{1}}{\sigma_{1}}\right)-\rho\left(\frac{a_{2}-\mu_{2}}{\sigma_{2}}\right)\right) c\right]-\Phi\left[\left(\left(\frac{b_{1}-\mu_{1}}{\sigma_{1}}\right)-\rho\left(\frac{a_{2}-\mu_{2}}{\sigma_{2}}\right)\right) c\right]\right] \\
& +\phi\left(\frac{b_{2}-\mu_{2}}{\sigma_{2}}\right)\left[\Phi\left[\left(\left(\frac{a_{1}-\mu_{1}}{\sigma_{1}}\right)-\rho\left(\frac{b_{2}-\mu_{2}}{\sigma_{2}}\right)\right) c\right]-\Phi\left[\left(\left(\frac{b_{2}-\mu_{2}}{\sigma_{2}}\right)-\rho\left(\frac{b_{2}-\mu_{2}}{\sigma_{2}}\right)\right) c\right]\right] \\
& -\rho \phi\left(\frac{a_{1}-\mu_{1}}{\sigma_{1}}\right)\left[\Phi\left[\left(\left(\frac{a_{2}-\mu_{2}}{\sigma_{2}}\right)-\rho\left(\frac{a_{1}-\mu_{1}}{\sigma_{1}}\right)\right) c\right]-\Phi\left[\left(\left(\frac{b_{2}-\mu_{2}}{\sigma_{2}}\right)-\rho\left(\frac{a_{1}-\mu_{1}}{\sigma_{1}}\right)\right) c\right]\right] \\
& \left.+\rho \phi\left(\frac{b_{1}-\mu_{1}}{\sigma_{1}}\right)\left[\Phi\left[\left(\left(\frac{a_{2}-\mu_{2}}{\sigma_{2}}\right)-\rho\left(\frac{b_{1}-\mu_{1}}{\sigma_{1}}\right)\right) c\right]-\Phi\left[\left(\left(\frac{b_{2}-\mu_{2}}{\sigma_{2}}\right)-\rho\left(\frac{b_{1}-\mu_{1}}{\sigma_{1}}\right)\right) c\right]\right]\right]
\end{aligned}
$$

and $c$ is given equation (3)

The Variance of $\mathrm{X}$ is

$\mathrm{V}(\mathrm{X})=\sigma_{1}^{2}\left[\mathrm{E}\left(\mathrm{Z}_{1}^{2}\right)\right]-\mathrm{A}^{2}\left(2 \sigma_{1}-1\right)$ 
where,

$$
\begin{aligned}
E\left(Z_{1}^{2}\right)=[ & \pi-\left(\frac{\mathrm{a}_{1}-\mu_{1}}{\sigma_{1}}\right) \phi\left(\frac{\mathrm{a}_{1}-\mu_{1}}{\sigma_{1}}\right)\left[\Phi\left[\left(\left(\frac{\mathrm{a}_{1}-\mu_{1}}{\sigma_{1}}\right)-\rho\left(\frac{\mathrm{a}_{1}-\mu_{1}}{\sigma_{1}}\right)\right) c\right]-\Phi\left[\left(\left(\frac{\mathrm{b}_{1}-\mu_{1}}{\sigma_{1}}\right)-\rho\left(\frac{\mathrm{a}_{1}-\mu_{1}}{\sigma_{1}}\right)\right) c\right]\right] \\
& +\left(\frac{\mathrm{b}_{1}-\mu_{1}}{\sigma_{1}}\right) \phi\left(\frac{\mathrm{b}_{1}-\mu_{1}}{\sigma_{1}}\right)\left[\Phi\left[\left(\left(\frac{\mathrm{a}_{1}-\mu_{1}}{\sigma_{1}}\right)-\rho\left(\frac{\mathrm{b}_{1}-\mu_{1}}{\sigma_{1}}\right)\right) c\right]-\Phi\left[\left(\left(\frac{\mathrm{b}_{1}-\mu_{1}}{\sigma_{1}}\right)-\rho\left(\frac{\mathrm{b}_{1}-\mu_{1}}{\sigma_{1}}\right)\right) c\right]\right] \\
& +\rho^{2}\left(\frac{\mathrm{a}_{1}-\mu_{1}}{\sigma_{1}}\right) \phi\left(\frac{\mathrm{a}_{1}-\mu_{1}}{\sigma_{1}}\right)\left[\Phi\left[\left(\left(\frac{\mathrm{a}_{1}-\mu_{1}}{\sigma_{1}}\right)-\rho\left(\frac{\mathrm{a}_{1}-\mu_{1}}{\sigma_{1}}\right)\right) c\right]-\Phi\left[\left(\left(\frac{\mathrm{b}_{1}-\mu_{1}}{\sigma_{1}}\right)-\rho\left(\frac{\mathrm{a}_{1}-\mu_{1}}{\sigma_{1}}\right)\right) c\right]\right] \\
& +c^{-1} \rho \phi\left(\frac{\mathrm{a}_{1}-\mu_{1}}{\sigma_{1}}\right)\left[\phi\left[\left(\left(\frac{\mathrm{a}_{1}-\mu_{1}}{\sigma_{1}}\right)-\rho\left(\frac{\mathrm{a}_{1}-\mu_{1}}{\sigma_{1}}\right)\right) c\right]-\phi\left[\left(\left(\frac{\mathrm{b}_{1}-\mu_{1}}{\sigma_{1}}\right)-\rho\left(\frac{\mathrm{a}_{1}-\mu_{1}}{\sigma_{1}}\right)\right) c\right]\right] \\
& +\rho^{2}\left(\frac{\mathrm{b}_{1}-\mu_{1}}{\sigma_{1}}\right) \phi\left(\frac{\mathrm{b}_{1}-\mu_{1}}{\sigma_{1}}\right)\left[\Phi\left[\left(\left(\left(\frac{\mathrm{a}_{1}-\mu_{1}}{\sigma_{1}}\right)-\rho\left(\frac{\mathrm{b}_{1}-\mu_{1}}{\sigma_{1}}\right)\right) c\right]-\Phi\left[\left(\left(\frac{\mathrm{b}_{1}-\mu_{1}}{\sigma_{1}}\right)-\rho\left(\frac{\mathrm{b}_{1}-\mu_{1}}{\sigma_{1}}\right)\right) c\right]\right]\right. \\
& \left.-c^{-1} \rho \phi\left(\frac{\mathrm{b}_{1}-\mu_{1}}{\sigma_{1}}\right)\left[\phi\left[\left(\left(\frac{\mathrm{a}_{1}-\mu_{1}}{\sigma_{1}}\right)-\rho\left(\frac{\mathrm{b}_{1}-\mu_{1}}{\sigma_{1}}\right)\right) c\right]-\phi\left[\left(\left(\frac{\mathrm{b}_{1}-\mu_{1}}{\sigma_{1}}\right)-\rho\left(\frac{\mathrm{b}_{1}-\mu_{1}}{\sigma_{1}}\right)\right) c\right]\right]\right]
\end{aligned}
$$

and $c, \mathrm{~A}$ are as given in equation (3)

The Variance of $\mathrm{Y}$ is

$$
\mathrm{V}(\mathrm{Y})=\sigma_{2}^{2}\left[\mathrm{E}\left(\mathrm{Z}_{2}^{2}\right)\right]-\mathrm{B}^{2}\left(2 \sigma_{2}-1\right)
$$

where,

$$
\begin{aligned}
\mathrm{E}\left(\mathrm{Z}_{2}^{2}\right)=[ & \left.\pi-\left(\frac{\mathrm{a}_{2}-\mu_{2}}{\sigma_{2}}\right) \phi\left(\frac{\mathrm{a}_{2}-\mu_{2}}{\sigma_{2}}\right)\left[\Phi\left[\left(\left(\frac{\mathrm{a}_{2}-\mu_{2}}{\sigma_{2}}\right)-\rho\left(\frac{\mathrm{a}_{2}-\mu_{2}}{\sigma_{2}}\right)\right) c\right]-\Phi\left[\left(\left(\frac{\mathrm{b}_{2}-\mu_{2}}{\sigma_{2}}\right)-\rho\left(\frac{\mathrm{a}_{2}-\mu_{2}}{\sigma_{2}}\right)\right)\right]\right]\right] \\
& +\left(\frac{\mathrm{b}_{2}-\mu_{2}}{\sigma_{2}}\right) \phi\left(\frac{\mathrm{b}_{2}-\mu_{2}}{\sigma_{2}}\right)\left[\Phi\left[\left(\left(\frac{\mathrm{a}_{2}-\mu_{2}}{\sigma_{2}}\right)-\rho\left(\frac{\mathrm{b}_{2}-\mu_{2}}{\sigma_{2}}\right)\right) c\right]-\Phi\left[\left(\left(\frac{\mathrm{b}_{2}-\mu_{2}}{\sigma_{2}}\right)-\rho\left(\frac{\mathrm{b}_{2}-\mu_{2}}{\sigma_{2}}\right)\right) c\right]\right] \\
& +\rho^{2}\left(\frac{\mathrm{a}_{2}-\mu_{2}}{\sigma_{2}}\right) \phi\left(\frac{\mathrm{a}_{2}-\mu_{2}}{\sigma_{2}}\right)\left[\Phi\left[\left(\left(\left(\frac{\mathrm{a}_{2}-\mu_{2}}{\sigma_{2}}\right)-\rho\left(\frac{\mathrm{a}_{2}-\mu_{2}}{\sigma_{2}}\right)\right) c\right]-\Phi\left[\left(\left(\frac{\mathrm{b}_{2}-\mu_{2}}{\sigma_{2}}\right)-\rho\left(\frac{\mathrm{a}_{2}-\mu_{2}}{\sigma_{2}}\right)\right) c\right]\right]\right. \\
& +c^{-1} \rho \phi\left(\frac{\mathrm{a}_{2}-\mu_{2}}{\sigma_{2}}\right)\left[\phi\left[\left(\left(\frac{\mathrm{a}_{2}-\mu_{2}}{\sigma_{2}}\right)-\rho\left(\frac{\mathrm{a}_{2}-\mu_{2}}{\sigma_{2}}\right)\right) c\right]-\phi\left[\left(\left(\frac{\mathrm{b}_{2}-\mu_{2}}{\sigma_{2}}\right)-\rho\left(\frac{\mathrm{a}_{2}-\mu_{2}}{\sigma_{2}}\right)\right) c\right]\right] \\
& +\rho^{2}\left(\frac{\mathrm{b}_{2}-\mu_{2}}{\sigma_{2}}\right) \phi\left(\frac{\mathrm{b}_{2}-\mu_{2}}{\sigma_{2}}\right)\left[\Phi\left[\left(\left(\left(\frac{\mathrm{a}_{2}-\mu_{2}}{\sigma_{2}}\right)-\rho\left(\frac{\mathrm{b}_{2}-\mu_{2}}{\sigma_{2}}\right)\right) c\right]-\Phi\left[\left(\left(\frac{\mathrm{b}_{2}-\mu_{2}}{\sigma_{2}}\right)-\rho\left(\frac{\mathrm{b}_{2}-\mu_{2}}{\sigma_{2}}\right)\right) c\right]\right]\right. \\
& \left.-c^{-1} \rho \phi\left(\frac{\mathrm{b}_{2}-\mu_{2}}{\sigma_{2}}\right)\left[\phi\left[\left(\left(\frac{\mathrm{a}_{2}-\mu_{2}}{\sigma_{2}}\right)-\rho\left(\frac{\mathrm{b}_{2}-\mu_{2}}{\sigma_{2}}\right)\right) c\right]-\phi\left[\left(\left(\frac{\mathrm{b}_{2}-\mu_{2}}{\sigma_{2}}\right)-\rho\left(\frac{\mathrm{b}_{2}-\mu_{2}}{\sigma_{2}}\right)\right) c\right]\right]\right]
\end{aligned}
$$

and $c$ and $\mathrm{B}$ are as given in equations (3) and (4) respectively. The Covariance of $(\mathrm{X}, \mathrm{Y})$ is

$$
\operatorname{COV}(\mathrm{X}, \mathrm{Y})=\sigma_{1} \sigma_{2} \mathrm{E}\left(\mathrm{Z}_{1}, \mathrm{Z}_{2}\right)-\mathrm{AB}\left[\sigma_{1}+\sigma_{2}-1\right]
$$

where,

$$
\begin{aligned}
\mathrm{E}\left(\mathbf{Z}_{1}, \mathbf{Z}_{2}\right)=[\rho & \left.-\rho\left(\frac{\mathbf{a}_{1}-\mu_{1}}{\sigma_{1}}\right) \phi\left(\frac{\mathbf{a}_{1}-\mu_{1}}{\sigma_{1}}\right)\left[\Phi\left[\left(\left(\frac{\mathbf{a}_{2}-\mu_{2}}{\sigma_{2}}\right)-\rho\left(\frac{\mathbf{a}_{1}-\mu_{1}}{\sigma_{1}}\right)\right) c\right]-\Phi\left[\left(\left(\frac{\mathbf{b}_{2}-\mu_{2}}{\sigma_{2}}\right)-\rho\left(\frac{\mathbf{a}_{1}-\mu_{1}}{\sigma_{1}}\right)\right)\right]\right]\right] \\
& +c^{-1} \phi\left(\frac{\mathbf{a}_{1}-\mu_{1}}{\sigma_{1}}\right)\left[\phi\left[\left(\left(\frac{\mathbf{a}_{2}-\mu_{2}}{\sigma_{2}}\right)-\rho\left(\frac{\mathbf{a}_{1}-\mu_{1}}{\sigma_{1}}\right)\right) c\right]-\phi\left[\left(\left(\frac{\mathbf{b}_{2}-\mu_{2}}{\sigma_{2}}\right)-\rho\left(\frac{\mathbf{a}_{1}-\mu_{1}}{\sigma_{1}}\right)\right) c\right]\right] \\
& +\rho\left(\frac{\mathbf{b}_{1}-\mu_{1}}{\sigma_{1}}\right) \phi\left(\frac{\mathbf{b}_{1}-\mu_{1}}{\sigma_{1}}\right)\left[\Phi\left[\left(\left(\frac{\mathbf{a}_{2}-\mu_{2}}{\sigma_{2}}\right)-\rho\left(\frac{\mathbf{b}_{1}-\mu_{1}}{\sigma_{1}}\right)\right) c\right]-\Phi\left[\left(\left(\frac{\mathbf{b}_{2}-\mu_{2}}{\sigma_{2}}\right)-\rho\left(\frac{\mathbf{b}_{1}-\mu_{1}}{\sigma_{1}}\right)\right) c\right]\right] \\
& -c^{-1} \phi\left(\frac{\mathbf{b}_{1}-\mu_{1}}{\sigma_{1}}\right)\left[\phi\left[\left(\left(\frac{\mathbf{a}_{2}-\mu_{2}}{\sigma_{2}}\right)-\rho\left(\frac{\mathbf{b}_{1}-\mu_{1}}{\sigma_{1}}\right)\right) c\right]-\phi\left[\left(\left(\frac{\mathbf{b}_{2}-\mu_{2}}{\sigma_{2}}\right)-\rho\left(\frac{\mathbf{b}_{1}-\mu_{1}}{\sigma_{1}}\right)\right) c\right]\right] \\
& \left.+\rho\left(\frac{\mathbf{a}_{2}-\mu_{2}}{\sigma_{2}}\right) \phi\left(\frac{\mathbf{a}_{2}-\mu_{2}}{\sigma_{2}}\right)\left[\Phi\left[\left(\left(\frac{\mathbf{a}_{1}-\mu_{1}}{\sigma_{1}}\right)-\rho\left(\frac{\mathbf{a}_{2}-\mu_{2}}{\sigma_{2}}\right)\right) c\right]-\Phi\left[\left(\left(\frac{\mathbf{b}_{1}-\mu_{1}}{\sigma_{1}}\right)-\rho\left(\frac{\mathbf{a}_{2}-\mu_{2}}{\sigma_{2}}\right)\right)\right]\right]\right] \\
& \left.+\rho\left(\frac{\mathbf{b}_{2}-\mu_{2}}{\sigma_{2}}\right) \phi\left(\frac{\mathbf{b}_{2}-\mu_{2}}{\sigma_{2}}\right)\left[\Phi\left[\left(\left(\frac{\mathbf{a}_{1}-\mu_{1}}{\sigma_{1}}\right)-\rho\left(\frac{\mathbf{b}_{2}-\mu_{2}}{\sigma_{2}}\right)\right) c\right]-\Phi\left[\left(\left(\frac{\mathbf{b}_{1}-\mu_{1}}{\sigma_{1}}\right)-\rho\left(\frac{\mathbf{b}_{2}-\mu_{2}}{\sigma_{2}}\right)\right) c\right]\right]\right]
\end{aligned}
$$

and c, A are as given in equation (3) and B is given in equation (4)

Since the entire image is a collection of regions, which are characterized by doubly truncated bivariate normal distribution, it can be characterized through a $K$-component finite doubly truncated bivariate Gaussian distribution and its probability density function is of the form

$h(x, y)=\sum_{i=1}^{K} \alpha_{i} g_{i}\left(x, y / \theta_{i}\right)$

where, $K$ is the number of regions, $\alpha_{i}>0$ are weights such that $\sum_{i=1}^{K} \alpha_{i}=1$ and $\theta=\left\{\mu_{1 i}, \mu_{2 i}, \sigma_{1 i}^{2}, \sigma_{2 i}^{2}, \rho_{i}\right\}$ is the parametric set. $g_{i}\left(x, y / \theta_{i}\right)$ is as given in equation (2) representing the probability density function of the $\mathrm{i}^{\text {th }}$ image region. $\alpha_{i}$ is the probability of occurrence of the $i^{\text {th }}$ component of the finite doubly truncated bivariate Gaussian mixture model (FDTBGMM) i.e., the probability that the feature belongs to the $\mathrm{i}^{\text {th }}$ image region.

The mean vector representing the entire image region is

$$
E(X, Y)=\left[\begin{array}{c}
\sum_{i=1}^{K} \alpha_{i} E_{i}(X) \\
\sum_{i=1}^{K} \alpha_{i} E_{i}(Y)
\end{array}\right]
$$

where, $E_{i}(X)$ and $E_{i}(Y)$ are as given in equations (3) and (4) for the $i^{\text {th }}$ image region.

\section{ESTIMATION OF THE MODEL PARAMETERS BY EM-ALGORITHM}

The likelihood function of bivariate observations $\left(x_{1}, y_{1}\right),\left(x_{2}, y_{2}\right),\left(x_{3}, y_{3}\right), \ldots,\left(x_{N}, y_{N}\right)$ drawn from an image with probability density function

$$
\begin{aligned}
& h(x, y ; \theta)=\sum_{i=1}^{K} \alpha_{i} g_{i}\left(x_{s}, y_{s} ; \theta\right) \text { is } \\
& \mathrm{L}(\theta)=\prod_{\boldsymbol{s}=\mathbf{1}}^{N}\left(\sum_{\boldsymbol{i}=\mathbf{1}}^{\boldsymbol{K}} \alpha_{\boldsymbol{i}} \boldsymbol{g}_{\boldsymbol{i}}\left(\boldsymbol{x}_{\boldsymbol{S}}, \boldsymbol{y}_{\boldsymbol{S}} ; \boldsymbol{\theta}\right)\right) \\
& =\prod_{s=1}^{N}\left(\sum_{i=1}^{K} \alpha_{i} \frac{\left.\exp \left\{\frac{-1}{2\left(1-\rho_{i}^{2}\right)}\left[\left(\frac{x_{s}-\mu_{1 i}}{\sigma_{1 i}}\right)^{2}-2 \rho_{i}\left(\frac{x_{s}-\mu_{1 i}}{\sigma_{1 i}}\right)\left(\frac{y_{s}-\mu_{2 i}}{\sigma_{2 i}}\right)+\left(\frac{y_{s}-\mu_{2 i}}{\sigma_{2 i}}\right)^{2}\right]\right\}\right)}{2 \pi \sqrt{1-\rho_{i}^{2}} \sigma_{1 i} \sigma_{2 i} \int_{b_{1} b_{2} b_{2}} f_{i}(x, y ; \theta) d x d y}\right)
\end{aligned}
$$

This implies

$$
\begin{aligned}
\log \mathrm{L}(\theta) & =\log \prod_{s=1}^{N}\left(\sum_{i=1}^{K} \alpha_{i} g_{i}\left(x_{s}, y_{s} ; \theta\right)\right. \\
& =\sum_{s=1}^{N} \log \left(\sum_{i=1}^{K} \alpha_{i} g_{i}\left(w_{s}, \theta\right)\right)
\end{aligned}
$$

The updated equations of EM-algorithm for estimating the model parameters are

$$
\begin{aligned}
\alpha_{k}^{(l+1)} & =\frac{1}{N} \sum_{s=1}^{N}\left[t_{k}\left(x_{s}, y_{s} ; \theta^{(l)}\right)\right] \\
& =\frac{1}{N} \sum_{s=1}^{N}\left(\frac{\alpha_{k}^{(l)} g_{k}\left(x_{s}, y_{s} ; \theta^{(l)}\right)}{\sum_{i=1}^{K} \alpha_{i}^{(l)} g_{i}\left(x_{s}, y_{s} ; \theta^{(l)}\right)}\right)
\end{aligned}
$$

where, $g_{k}\left(x_{s}, y_{s} ; \theta^{(l)}\right)$ is as given in equation (2).

The updated equation of $\mu_{1 k}$ at $(l+1)^{\text {th }}$ iteration is,

$\mu_{1 k}^{(l+1)} \sum_{s=1}^{N} t_{k}\left(x_{s}, y_{s} ; \theta^{(l)}\right)-\sum_{s=1}^{N} t_{k}\left(x_{s}, y_{s} ; \theta^{(l)}\right) x_{s}+\sum_{s=1}^{N} t_{k}\left(x_{s}, y_{s} ; \theta^{(l)}\right) \sigma_{1 k}^{(l)}\left[\frac{\rho_{k}^{(l)}\left(y_{s}-\mu_{2 k}^{(l)}\right)}{\sigma_{2 k}^{(l)}}+\left[\mathrm{A}-\rho_{k}^{(l)} \mathrm{B}\right]\right]=0$ 
where,

$$
\begin{aligned}
& t_{k}\left(x_{S}, y_{S} ; \theta^{(l)}\right)=\frac{\alpha_{k}^{(l)} g_{k}\left(x_{S}, y_{s} ; \theta^{(l)}\right)}{h\left(x_{s}, y_{S} ; \theta^{(l)}\right)}=\frac{\alpha_{k}^{(l)} g_{k}\left(x_{s}, y_{S} ; \theta^{(l)}\right)}{\sum_{i=1}^{K} \alpha_{i}^{(l)} g_{i}\left(x_{S}, y_{S} ; \theta^{(l)}\right)}, \\
& g_{k}\left(x_{s}, y_{S} ; \theta^{(l)}\right)=\frac{\exp \left\{\frac{-1}{2\left(1-\rho_{k}^{2}\right)}\left[\left(\frac{x_{s}-\mu_{1 k}}{\sigma_{1 k}}\right)^{2}-2 \rho_{k}\left(\frac{x_{s}-\mu_{1 k}}{\sigma_{1 k}}\right)\left(\frac{y_{s}-\mu_{2 k}}{\sigma_{2 k}}\right)+\left(\frac{y_{s}-\mu_{2 k}}{\sigma_{2 k}}\right)^{2}\right]\right\}}{2 \pi \sigma_{1 k} \sigma_{2 k} \sqrt{1-\rho_{k}^{2}} \int_{b_{1}}^{a_{1} a_{2}} \int_{b_{2}} f_{k}\left(x, y ; \theta^{(l)}\right) d x d y},
\end{aligned}
$$

$\mathrm{A}$ and $\mathrm{B}$ are as given in equations (3) and (4) respectively.

Similarly the updated equation of $\mu_{2 k}$ at $(l+1)^{\text {th }}$ iteration is $\mu_{2 k}^{(l+1)} \sum_{s=1}^{N} t_{k}\left(x_{s}, y_{s} ; \theta^{(l)}\right)-\sum_{s=1}^{N} t_{k}\left(x_{s}, y_{s} ; \theta^{(l)}\right) y_{s}+\sum_{s=1}^{N} t_{k}\left(x_{s}, y_{s} ; \theta^{(l)}\right) \sigma_{2 k}^{(l)}\left[\frac{\rho_{k}^{(l)}\left(x_{s}-\mu_{1 k}^{(l)}\right)}{\sigma_{k}^{(l)}}+\left[\mathrm{B}-\rho_{k}^{(l)} \mathrm{A}\right]\right]=0$

where, $t_{k}\left(x_{s}, y_{s} ; \theta^{(l)}\right)$ is given in equation (13), $\mathrm{A}$ and $\mathrm{B}$ are as given in equations (3) and (4) respectively.

The updated equations for $\sigma_{1 k}^{2}$ at $(l+1)^{\text {th }}$ iteration is

$$
\sum_{s=1}^{N} t_{k}\left(x_{s}, y_{s} ; \theta^{(l)}\right)\left[\left[\left(\frac{x_{s}-\mu_{1 k}^{(l)}}{\sigma_{1 k}^{(l+1)}}\right)^{2}-\frac{\rho_{k}\left(x_{s}-\mu_{1 k}^{(l)}\right)\left(y_{s}-\mu_{2 k}^{(l)}\right)}{\sigma_{1 k}^{(l+1)} \sigma_{2 k}^{(l)}}\right]-D+\rho_{k}^{(l)} E\right]=0
$$

where, $t_{k}\left(x_{s}, y_{s} ; \theta^{(l)}\right)$ is given in equation (13),

$$
\begin{aligned}
D=\pi \sigma_{1 k} \sigma_{2 k} & +\sigma_{1 k} \sigma_{2 k} c^{-1} \rho_{k}\left[\phi\left(\mathrm{A}_{1}\right)\left[\phi\left[\left(\left(\mathrm{A}_{1}\right)-\rho_{k}\left(\mathrm{~A}_{1}\right)\right) c\right]-\phi\left[\left(\left(\mathrm{B}_{1}\right)-\rho_{k}\left(\mathrm{~A}_{1}\right)\right) c\right]\right]\right. \\
& \left.-\phi\left(\mathrm{B}_{1}\right)\left[\phi\left[\left(\left(\mathrm{A}_{1}\right)-\rho_{k}\left(\mathrm{~B}_{1}\right)\right) c\right]-\phi\left[\left(\left(\mathrm{B}_{1}\right)-\rho_{k}\left(\mathrm{~B}_{1}\right)\right) c\right]\right]\right] \\
& +\sigma_{2 k}\left(\rho_{k}^{2}-1\right)\left(\mathrm{a}_{1}-\mu_{1 k}\right) \phi\left(\mathrm{A}_{1}\right)\left[\Phi\left[\left(\left(\mathrm{A}_{1}\right)-\rho_{k}\left(\mathrm{~A}_{1}\right)\right) c\right]-\Phi\left[\left(\left(\mathrm{B}_{1}\right)-\rho_{k}\left(\mathrm{~A}_{1}\right)\right) c\right]\right] \\
& +\sigma_{2 k}\left(\rho_{k}^{2}+1\right)\left(b_{1}-\mu_{1 k}\right) \phi\left(\mathrm{B}_{1}\right)\left[\Phi\left[\left(\left(\mathrm{A}_{1}\right)-\rho_{k}\left(\mathrm{~B}_{1}\right)\right) c\right]-\Phi\left[\left(\left(\mathrm{B}_{1}\right)-\rho_{k}\left(\mathrm{~B}_{1}\right)\right) c\right]\right]
\end{aligned}
$$

and

$$
\begin{gathered}
E=\rho_{k} \pi \sigma_{1 k} \sigma_{2 k}+\sigma_{1 k} \sigma_{2 k} c^{-1}\left[\phi\left(\mathrm{A}_{1}\right)\left[\phi\left[\left(\left(\mathrm{A}_{2}\right)-\rho_{k}\left(\mathrm{~A}_{1}\right)\right) c\right]-\phi\left[\left(\left(\mathrm{B}_{2}\right)-\rho_{k}\left(\mathrm{~A}_{1}\right)\right) c\right]\right]\right. \\
\left.-\phi\left(\mathrm{B}_{1}\right)\left[\phi\left[\left(\left(\mathrm{A}_{2}\right)-\rho_{k}\left(\mathrm{~B}_{1}\right)\right) c\right]-\phi\left[\left(\left(\mathrm{B}_{2}\right)-\rho_{k}\left(\mathrm{~B}_{1}\right)\right) c\right]\right]\right] \\
+\rho_{k} \sigma_{1 k}\left[\left(\mathrm{a}_{2}-\mu_{2 k}\right) \phi\left(\mathrm{A}_{2}\right)\left[\Phi\left[\left(\left(\mathrm{A}_{1}\right)-\rho_{k}\left(\mathrm{~A}_{2}\right)\right) c\right]-\Phi\left[\left(\left(\mathrm{B}_{1}\right)-\rho_{k}\left(\mathrm{~A}_{2}\right)\right) c\right]\right]\right. \\
+\left(b_{2}-\mu_{2 k}\right) \phi\left(\mathrm{B}_{2}\right)\left[\Phi\left[\left(\left(\mathrm{A}_{1}\right)-\rho_{k}\left(\mathrm{~B}_{2}\right)\right) c\right]-\Phi\left[\left(\left(\mathrm{B}_{1}\right)-\rho_{k}\left(\mathrm{~B}_{2}\right)\right) c\right]\right] \\
+\rho_{k} \sigma_{2 k}\left[\left(\mathrm{~b}_{1}-\mu_{1 k}\right) \phi\left(\mathrm{B}_{1}\right)\left[\Phi\left[\left(\left(\mathrm{A}_{2}\right)-\rho_{k}\left(\mathrm{~B}_{1}\right)\right) c\right]-\Phi\left[\left(\left(\mathrm{B}_{2}\right)-\rho_{k}\left(\mathrm{~B}_{1}\right)\right) c\right]\right]\right. \\
+\left(a_{1}-\mu_{1 k}\right) \phi\left(\mathrm{A}_{1}\right)\left[\Phi\left[\left(\left(\mathrm{A}_{2}\right)-\rho_{k}\left(\mathrm{~A}_{1}\right)\right) c\right]-\Phi\left[\left(\left(\mathrm{B}_{2}\right)-\rho_{k}\left(\mathrm{~A}_{1}\right)\right) c\right]\right],
\end{gathered}
$$

Similarly the updated equation for $\sigma_{2 k}^{2}$ at $(l+1)^{\text {th }}$ iteration is

$$
\sum_{s=1}^{N} t_{k}\left(x_{s}, y_{s} ; \theta^{(l)}\right)\left[\left[\left(\frac{y_{s}-\mu_{2 k}^{(l)}}{\sigma_{2 k}^{(l+1)}}\right)^{2}-\frac{\rho_{k}\left(x_{s}-\mu_{1 k}^{(l)}\right)\left(y_{s}-\mu_{2 k}^{(l)}\right)}{\sigma_{1 k}^{(l)} \sigma_{2 k}^{(l+1)}}\right]-G+\rho_{k}^{(l)} E\right]=0
$$

where, $t_{k}\left(x_{s}, y_{s} ; \theta^{(l)}\right)$ is given in equation (13), $E$ is given in equation (15) and

$$
\begin{array}{r}
G=\pi \sigma_{1 k} \sigma_{2 k}+\sigma_{1 k} \sigma_{2 k} c^{-1} \rho_{k}\left[\phi\left(\mathrm{A}_{2}\right)\left[\phi\left[\left(\left(\mathrm{A}_{2}\right)-\rho_{k}\left(\mathrm{~A}_{2}\right)\right) c\right]-\phi\left[\left(\left(\mathrm{B}_{2}\right)-\rho_{k}\left(\mathrm{~A}_{2}\right)\right) c\right]\right]\right. \\
\left.-\phi\left(\mathrm{B}_{2}\right)\left[\phi\left[\left(\left(\mathrm{A}_{2}\right)-\rho_{k}\left(\mathrm{~B}_{2}\right)\right) c\right]-\phi\left[\left(\left(\mathrm{B}_{2}\right)-\rho_{k}\left(\mathrm{~B}_{2}\right)\right) c\right]\right]\right] \\
+\sigma_{1 k}\left(\rho_{k}^{2}-1\right)\left(\mathrm{a}_{2}-\mu_{2 k}\right) \phi\left(\mathrm{A}_{2}\right)\left[\Phi\left[\left(\left(\mathrm{A}_{2}\right)-\rho_{k}\left(\mathrm{~A}_{2}\right)\right) c\right]-\Phi\left[\left(\left(\mathrm{B}_{2}\right)-\rho_{k}\left(\mathrm{~A}_{2}\right)\right) c\right]\right] \\
+\sigma_{1 k}\left(\rho_{k}^{2}+1\right)\left(b_{2}-\mu_{2 k}\right) \phi\left(\mathrm{B}_{2}\right)\left[\Phi\left[\left(\left(\mathrm{A}_{2}\right)-\rho_{k}\left(\mathrm{~B}_{2}\right)\right) c\right]-\Phi\left[\left(\left(\mathrm{B}_{2}\right)-\rho_{k}\left(\mathrm{~B}_{2}\right)\right) c\right]\right]
\end{array}
$$

The updated equation for estimating $\rho_{k}$ is

$$
\begin{aligned}
\sum_{s=1}^{N} t_{k}\left(x_{s}, y_{s} ; \theta^{(l)}\right) & {\left[-\frac{\rho_{k}}{\left(1-\rho_{k}^{2}\right)^{2}}\left[\left(\frac{x_{s}-\mu_{1 k}}{\sigma_{1 k}}\right)^{2}+\left(\frac{y_{s}-\mu_{2 k}}{\sigma_{2 k}}\right)^{2}\right]\right.} \\
& \left.-\frac{1+\rho_{k}^{2}}{\left(1-\rho_{k}^{2}\right)^{2}}\left[\left(\frac{x_{s}-\mu_{1 k}}{\sigma_{1 k}}\right)\left(\frac{y_{s}-\mu_{2 k}}{\sigma_{2 k}}\right)\right]+\frac{\rho_{k}(D+F)}{\left(1-\rho_{k}^{2}\right)^{2}}+\frac{\left(1+\rho_{k}^{2}\right) E}{\left(1-\rho_{k}^{2}\right)^{2}}\right]=0
\end{aligned}
$$

where, $t_{k}\left(x_{s}, y_{s} ; \theta^{(l)}\right)$ is given in equation (13), $D, E$ are given in equation (15)

and

$$
\begin{array}{r}
F=\pi \sigma_{1 k} \sigma_{2 k}-\sigma_{1 k}\left[\left(\mathrm{a}_{2}-\mu_{2 \mathrm{k}}\right) \phi\left(\mathrm{A}_{2}\right)\left[\Phi\left[\left(\left(\mathrm{A}_{2}\right)-\rho_{k}\left(\mathrm{~A}_{2}\right)\right) c\right]-\Phi\left[\left(\left(\mathrm{B}_{2}\right)-\rho_{k}\left(\mathrm{~A}_{2}\right)\right) c\right]\right]\right. \\
\left.-\left(\mathrm{b}_{2}-\mu_{2 \mathrm{k}}\right) \phi\left(\mathrm{B}_{2}\right)\left[\Phi\left[\left(\left(\mathrm{A}_{2}\right)-\rho_{k}\left(\mathrm{~B}_{2}\right)\right) c\right]-\Phi\left[\left(\left(\mathrm{B}_{2}\right)-\rho_{k}\left(\mathrm{~B}_{2}\right)\right) c\right]\right]\right] \\
+\sigma_{1 k} \rho_{k}^{2}\left[\left(\mathrm{a}_{2}-\mu_{2 \mathrm{k}}\right) \phi\left(\mathrm{A}_{2}\right)\left[\Phi\left[\left(\left(\mathrm{A}_{2}\right)-\rho_{k}\left(\mathrm{~A}_{2}\right)\right) c\right]-\Phi\left[\left(\left(\mathrm{B}_{2}\right)-\rho_{k}\left(\mathrm{~A}_{2}\right)\right) c\right]\right]\right. \\
\left.+\left(\mathrm{b}_{2}-\mu_{2 \mathrm{k}}\right) \phi\left(\mathrm{B}_{2}\right)\left[\Phi\left[\left(\left(\mathrm{A}_{2}\right)-\rho_{k}\left(\mathrm{~B}_{2}\right)\right) c\right]-\Phi\left[\left(\left(\mathrm{B}_{2}\right)-\rho\left(\mathrm{B}_{2}\right)\right) c\right]\right]\right] \\
+\sigma_{1 k} \sigma_{2 k} \rho_{k} c^{-1}\left[\phi\left(\mathrm{A}_{2}\right)\left[\phi\left[\left(\left(\mathrm{A}_{2}\right)-\rho_{k}\left(\mathrm{~A}_{2}\right)\right) c\right]-\phi\left[\left(\left(\mathrm{B}_{2}\right)-\rho_{k}\left(\mathrm{~A}_{2}\right)\right) c\right]\right]\right. \\
\left.-\phi\left(\mathrm{B}_{2}\right)\left[\phi\left[\left(\left(\mathrm{A}_{2}\right)-\rho_{k}\left(\mathrm{~B}_{2}\right)\right) c\right]-\phi\left[\left(\left(\mathrm{B}_{2}\right)-\rho_{k}\left(\mathrm{~B}_{2}\right)\right) c\right]\right]\right]
\end{array}
$$

where,

$$
\mathrm{A}_{1}=\frac{a_{1}-\mu_{1 k}}{\sigma_{1 k}}, \mathrm{~A}_{2}=\frac{a_{2}-\mu_{2 k}}{\sigma_{2 k}}, \mathrm{~B}_{1}=\frac{b_{1}-\mu_{1 k}}{\sigma_{1 k}} \text {, and } \mathrm{B}_{2}=\frac{b_{2}-\mu_{2 k}}{\sigma_{2 k}}
$$

Solving equations (13), (14),(15),(16) and (17) iteratively using numerical methods we get the revised estimates of $\mu_{1 k}, \mu_{2 k}, \sigma_{1 k}^{2}$,

$$
\sigma_{2 k}^{2} \text { and } \rho_{k} \text {. }
$$

\section{INITIALIZATION OF THE PARAMETERS BY K-MEANS}

The efficiency of the EM-algorithm in estimating the parameters is heavily dependent on the number of regions in the image. The number of mixture components initially taken for $K$-means algorithm is by plotting the histogram of the pixel intensities of the whole image. The number of peaks in the histogram can be taken as the initial value of the number of regions $K$. Usually the mixing parameter $\alpha_{k}$ and the region parameters $\mu_{1 k}, \mu_{2 k}, \sigma_{1 k}^{2}, \sigma_{2 k}^{2}$, $\rho_{k}$ are unknown. A commonly used method in initialization is by drawing a random sample in the entire image data (Mclanchlan G. and Krishnan T. (1997), Mclanchlan G. and Peel D. (2000)). This method perform well only when the sample size is large, and the computation time is heavily increased. When the sample size is small it is likely that some small regions may not be sampled. To overcome this problem, we use $K$-means algorithm (Rose H. Turi (2001)) to divide the whole image into various homogeneous regions. 
After determining the value of $K$ (number of regions), and dividing the feature vectors of image pixels in the whole region into $K$ regions. We obtain the initial estimates of the parameters $\mu_{1 k}$, $\mu_{2 k}, \sigma_{1 k}^{2}, \sigma_{2 k}^{2}, \rho_{k}$ and $\alpha_{k}$ for the $K^{\text {th }}$ region and with the movement method of estimators given by Bengt Muthen (1990) for Truncated Bivariate Normal Distribution with initial parameters as $\alpha_{\mathrm{i}}=1 / K$ for $i=1,2, \ldots, K$

$\mu_{1 k}=\left(\bar{x}_{k}-b_{1}\right)$, where, $b_{1}$ is the left truncated point for Hue and $\bar{x}_{1 k}$ is the $K^{\text {th }}$ region sample mean of the Hue angle.

$\mu_{2 k}=\left(\bar{y}_{k}-b_{2}\right)$, where, $b_{2}$ is the left truncated point for Saturation and $\bar{y}_{1 k}$ is the $k^{\text {th }}$ region sample mean of the Saturation.

$\sigma_{1 k}=\mathrm{S}_{1 k}$ (Sample Standard Deviation of the $K^{\mathrm{th}}$ segment of Hue) $\sigma_{2 k}=S_{2 k}$ (Sample Standard Deviation of the $K^{\text {th }}$ segment of Saturation)

$\rho_{k}$ is the correlation coefficient between Hue and Saturation of the $k^{\text {th }}$ image region.

Substituting these values as the initial estimates, we obtain the refined estimates of the parameters by using the EM-algorithm.

\section{SEGMENTATION ALGORITHM}

In this section we present the image segmentation algorithm. After refining the parameters the prime step in image segmentation is allocating the pixels to the segments of the image. This operation is performed by Segmentation Algorithm. The image segmentation algorithm consists of four steps.

Step 1) Plot the histogram of the whole image.

Step 2) Obtain the initial estimates of the model parameters using $\mathrm{K}$-means algorithm and moment estimators as discussed in section 4.

Step 3) Obtain the refined estimates of the model parameters by using the EM-algorithm with the updated equations given by (12), (13), (14), (15),(16) and (17) respectively in section 3.

S tep 4) Assign each pixel into the corresponding $\mathrm{j}^{\text {th }}$ region (segment) according to the component Maximum likelihood of the $\mathrm{j}^{\text {th }}$ component $L_{j}$. That is,

$$
L_{j}=\max _{j \in k}\left\{\frac{\exp \left\{\frac{-1}{2\left(1-\rho_{k}^{2}\right)}\left[\left(\frac{x_{s}-\mu_{1 k}}{\sigma_{1 k}}\right)^{2}-2 \rho_{k}\left(\frac{x_{s}-\mu_{1 k}}{\sigma_{1 k}}\right)\left(\frac{y_{s}-\mu_{2 k}}{\sigma_{2 k}}\right)+\left(\frac{y_{s}-\mu_{2 k}}{\sigma_{2 k}}\right)^{2}\right]\right\}}{2 \pi \sigma_{1 k} \sigma_{2 k} \sqrt{1-\rho_{k}^{2}} \int_{b_{1}}^{a_{1}} \int_{b_{2}}^{a_{2}} f_{k}(x, y ; \theta) d x d y}\right\}
$$

\section{EXPERIMENTAL RESULTS}

In order to evaluate the performance of the proposed image segmentation algorithm with bivariate Truncated Gaussian Mixture model it has been applied to six images namely, OSTRICH, POT, TOWER, BEARS, DEER and BIRD are taken from Berkeley images dataset. (http://www.eecs.berkeley.edu/Research/Projects/ $\mathrm{CS} /$ vision/bsds/BSDS300/html/dataset/images.html). The pixels feature vector of the whole image are taken as input for image segmentation. It is assumed that in each image region the feature vector follows a Doubly Truncated Bivariate Gaussian distribution with different parameters. The number of segments in each of the six images

considered for experimentation is determined by the histogram of pixel intensities. The histograms of the pixel intensities of the six images are shown in Figure 1.

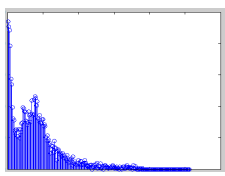

OSTRICH

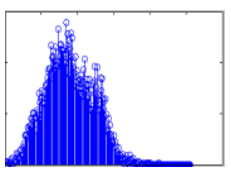

BEARS

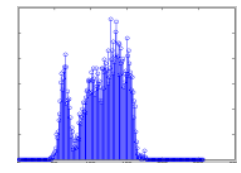

POT

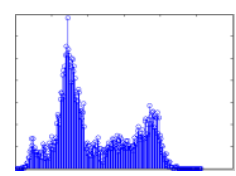

DEER

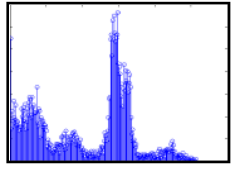

TOWER

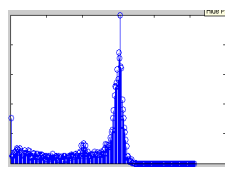

BIRD
Figure 1: Histograms of the Images

From the histograms given in Figure 1, the initial estimates of the number of regions in each image (' $K$ ') are obtained and given in Table 1.

Table 1: Initial Estimates of $K$

\begin{tabular}{|c|c|c|c|c|c|c|}
\hline IMAGE & OSTRICH & POT & TOWER & BEARS & DEER & BIRD \\
\hline Estimate of K & $\mathbf{2}$ & $\mathbf{3}$ & $\mathbf{4}$ & $\mathbf{2}$ & $\mathbf{4}$ & $\mathbf{3}$ \\
\hline
\end{tabular}

From Table 1, we observe that the images OSTRICH and BEARS have two segments, images POT and BIRD have three segments each and images TOWER and DEER has four segments. After assigning these initial values of $K$ to each image data set, the $K$ means algorithm is performed. The initial values of the model parameters $\mu_{1 i}, \mu_{2 i}, \sigma_{1 i}^{2}, \sigma_{2 i}^{2}, \rho_{i}$ and $\alpha_{i}$ for $\mathrm{i}=1,2, \ldots, K$ for each image region of the images are computed by using the method given in section (3.4). Using these initial estimates, the refined estimates of the model parameters for each image region are obtained by using EM-algorithm given in section 3.3. The computed values of the initial estimates and the final estimates of the model parameters $\mathrm{K}, \mu_{1 i}, \mu_{2 i}, \sigma_{1 i}^{2}, \sigma_{2 i}^{2}, \rho_{i}$ and $\alpha_{i}$ for $\mathrm{i}=1,2 \ldots, K$ for each image are obtained and presented in Tables 2.a, 2.b, 2.c, 2.d, 2.e and 2.f. for different images .

Table-2.a : Estimated Values of The Parameters For OS TRICH Image

\begin{tabular}{|c|c|c|c|c|c|}
\hline \multicolumn{4}{|c|}{ Estimation of Initial Parameters } & \multirow{2}{*}{\multicolumn{2}{|c|}{$\begin{array}{c}\begin{array}{c}\text { Estimation of Final Parameters } \\
\text { by EM-Algorithm }\end{array} \\
\text { Number of Image } \\
\text { Regions }(\boldsymbol{K}=2)\end{array}$}} \\
\hline \multirow{2}{*}{\multicolumn{2}{|c|}{ Regions(i) }} & \multicolumn{2}{|c|}{$\begin{array}{l}\text { Number of Image } \\
\text { Regions }(K=2)\end{array}$} & & \\
\hline & & 1 & 2 & 1 & 2 \\
\hline Weights & $\alpha_{i}$ & $1 / 2$ & $1 / 2$ & 0.2627 & 0.7373 \\
\hline \multirow{2}{*}{ Means } & $\mu_{1 i}$ & & & 0.1524 & 03100 \\
\hline & $\mu_{2 i}$ & 03321 & 0.7613 & 0.4497 & 0.4195 \\
\hline \multirow{2}{*}{ Variances } & $\sigma_{1 i}^{2}$ & 0.0016 & 0.0004 & 0.0306 & 0.0342 \\
\hline & $\sigma_{2 i}^{2}$ & 0.0126 & 0.0207 & 0.0255 & 09525 \\
\hline $\begin{array}{l}\text { Correlation } \\
\text { Coefficient }\end{array}$ & $\rho_{i}$ & -0.4310 & 0.6996 & 0.4003 & 0.1413 \\
\hline
\end{tabular}


Table-2.b : Estimated Values of The Parameters For POT Image

\begin{tabular}{|c|c|c|c|c|c|c|c|}
\hline Est & ation $\mathrm{s}$ & f Initia & 1 Param & & Estima & $\begin{array}{l}1 \text { of Fir } \\
\text { EM-Al }\end{array}$ & $\begin{array}{l}\text { ameters } \\
\mathrm{m}\end{array}$ \\
\hline Regions (i) & & & $\begin{array}{l}\text { Numbe } \\
\text { Regio }\end{array}$ & $\begin{array}{l}\text { Image } \\
K=3)\end{array}$ & & $\begin{array}{l}\text { ther o } \\
\text { ions }\end{array}$ & \\
\hline & & 1 & 2 & 3 & 1 & 2 & 3 \\
\hline Weights & $\alpha_{i}$ & $1 / 3$ & $1 / 3$ & $1 / 3$ & 0.4646 & 0.2266 & 0.3088 \\
\hline Means & $\mu_{1 i}$ & 05532 & 0.4946 & 0.1517 & 05602 & 0.6073 & 05686 \\
\hline & $\mu_{2 i}$ & 02168 & 0.1125 & 0.1219 & 0.3367 & 05654 & 0.4251 \\
\hline Variances & $\sigma_{1 i}^{2}$ & 0.0004 & 0.0027 & 0.0029 & 0.1629 & 0.4147 & 0.9292 \\
\hline & $\sigma_{2 i}^{2}$ & 0.0008 & 0.0018 & 0.0035 & 0.5247 & 1.4166 & 0.3976 \\
\hline $\begin{array}{l}\text { Correlation } \\
\text { Coefficient }\end{array}$ & $\rho_{i}$ & 0.1666 & 03570 & -0.7230 & -0.1097 & 0.0596 & -0.7175 \\
\hline $\begin{array}{l}\text { Minimum Hu } \\
\text { Minimum Sat }\end{array}$ & $\begin{array}{l}\text { Value } \\
\text { ration }\end{array}$ & $\begin{array}{l}\left(\mathbf{b}_{1}\right)= \\
\text { Value }\end{array}$ & $\begin{array}{l}\mathbf{0} \\
\left(\mathbf{b}_{2}\right)=\end{array}$ & $\begin{array}{l}\text { Maxim } \\
\text { Maxim }\end{array}$ & $\begin{array}{l}\text { Aue Va } \\
\text { Saturat }\end{array}$ & $\begin{array}{l}\left(a_{1}\right)= \\
\text { Valu }\end{array}$ & $\begin{array}{l}54 \\
=0.345\end{array}$ \\
\hline
\end{tabular}

Table-2.c: Estimated Values of The Parameters For TOW ER Image

\begin{tabular}{|c|c|c|c|c|c|c|c|c|c|}
\hline \multicolumn{6}{|c|}{ Estimation of Initial Parameters } & \multirow{2}{*}{\multicolumn{4}{|c|}{$\begin{array}{c}\begin{array}{c}\text { Estimation of Final Parameters } \\
\text { by EM-Algorithm }\end{array} \\
\text { Number of Image } \\
\text { Regions }(K=4)\end{array}$}} \\
\hline \multirow{2}{*}{\multicolumn{2}{|c|}{ Regions(i) }} & \multicolumn{4}{|c|}{$\begin{array}{l}\text { Number of Image } \\
\text { Regions }(K=4)\end{array}$} & & & & \\
\hline & & 1 & 2 & 3 & 4 & 1 & 2 & 3 & 4 \\
\hline Weights & $\alpha_{i}$ & $1 / 4$ & $1 / 4$ & $1 / 4$ & $1 / 4$ & 0.1999 & 0.1523 & 0.1872 & 0.4606 \\
\hline Means & $\begin{array}{l}\mu_{1 i} \\
\mu_{2 i}\end{array}$ & $\begin{array}{l}0.1519 \\
0.1937\end{array}$ & $\begin{array}{r}0.5699 \\
0.2789\end{array}$ & $\begin{array}{l}0.1505 \\
0.6176\end{array}$ & $\begin{array}{l}0.5738 \\
0.7724\end{array}$ & $\begin{array}{l}0.9545 \\
0.3257\end{array}$ & $\begin{array}{l}0.6581 \\
0.8931\end{array}$ & $\begin{array}{l}0.1677 \\
05290\end{array}$ & $\begin{array}{l}0.5905 \\
0.1014\end{array}$ \\
\hline Variances & $\begin{array}{c}\sigma_{1 i}^{2} \\
2 \\
\sigma_{2 i}^{2}\end{array}$ & $\begin{array}{l}0.0033 \\
0.0148\end{array}$ & $\begin{array}{l}0.0073 \\
0.0148\end{array}$ & $\begin{array}{l}0.0011 \\
0.0291\end{array}$ & $\begin{array}{l}0.0006 \\
0.0059\end{array}$ & $\begin{array}{l}1.1972 \\
0.0499\end{array}$ & $\begin{array}{l}0.0192 \\
0.3421\end{array}$ & $\begin{array}{l}0.2460 \\
0.6459\end{array}$ & $\begin{array}{l}0.0109 \\
1.6882\end{array}$ \\
\hline $\begin{array}{c}\text { Correlation } \\
\text { Coefficient }\end{array}$ & $\rho_{i}$ & -0.1561 & 0.0259 & 0.0386 & -0.1086 & -0.5562 & -0.8871 & -0.5036 & -0.5998 \\
\hline
\end{tabular}

Table-2.d : Estimated Values of The Parameters For BEARS Image

\begin{tabular}{|c|c|c|c|c|c|}
\hline \multicolumn{4}{|c|}{ Estimation of Initial Parameters } & \multirow{2}{*}{\multicolumn{2}{|c|}{$\begin{array}{c}\begin{array}{c}\text { Estimation of Final Parameters } \\
\text { by E M-Algorithm }\end{array} \\
\text { Number of Image } \\
\text { Regions }(K=2)\end{array}$}} \\
\hline \multicolumn{2}{|l|}{ Regions(i) } & \multicolumn{2}{|c|}{$\begin{array}{l}\text { Number of Image } \\
\text { Regions }(K=2)\end{array}$} & & \\
\hline & & 1 & 2 & 1 & 2 \\
\hline Weights & $\alpha_{i}$ & $1 / 2$ & $1 / 2$ & 0.4531 & 05469 \\
\hline \multirow[t]{2}{*}{ Means } & $\mu_{1 i}$ & & & & \\
\hline & $\mu_{2 i}$ & 0.4532 & 0.2600 & 09112 & 0.9711 \\
\hline \multirow{2}{*}{ Variances } & $\sigma_{1 i}^{2}$ & 0.0027 & 0.0154 & 0.0177 & 0.6701 \\
\hline & $\sigma_{2 i}^{2}$ & 0.0129 & 0.0170 & 0.6701 & 03924 \\
\hline $\begin{array}{l}\text { Correlation } \\
\text { Coefficient }\end{array}$ & $\rho_{i}$ & 0.2044 & -0.6378 & -0.4916 & 03233 \\
\hline $\begin{array}{l}\text { Minimum Hue } \\
\text { Minimum Satur }\end{array}$ & & & & isaturatic & $=1.0000$ \\
\hline
\end{tabular}

Table-2.e: Estimated Values of The Paramete rs For DEER Image

\begin{tabular}{|c|c|c|c|c|c|c|c|c|c|}
\hline \multicolumn{6}{|c|}{ Estimation of Initial Parameters } & \multirow{2}{*}{\multicolumn{4}{|c|}{$\begin{array}{c}\begin{array}{c}\text { Estimation of Final Parameters } \\
\text { by EM-Algorithm }\end{array} \\
\text { Number of Image } \\
\text { Regions }(K=4) \\
\end{array}$}} \\
\hline \multirow{2}{*}{\multicolumn{2}{|c|}{ Regions(i) }} & \multicolumn{4}{|c|}{$\begin{array}{c}\text { Number of Image } \\
\text { Regions }(K=4)\end{array}$} & & & & \\
\hline & & 1 & 2 & 3 & $\mathbf{4}$ & 1 & 2 & 3 & 4 \\
\hline Weights & $\alpha_{i}$ & $1 / 4$ & $1 / 4$ & $1 / 4$ & $1 / 4$ & 0.0704 & 0.4769 & 0.2771 & 0.1756 \\
\hline Means & $\begin{array}{l}\mu_{1 i} \\
\mu_{2 i}\end{array}$ & $\begin{array}{l}0.1299 \\
0.6989\end{array}$ & $\begin{array}{l}0.1144 \\
0.4560\end{array}$ & $\begin{array}{l}0.2324 \\
0.2354\end{array}$ & $\begin{array}{l}0.3016 \\
0.1262\end{array}$ & $\begin{array}{l}0.1779 \\
05062\end{array}$ & $\begin{array}{l}0.4502 \\
0.5224\end{array}$ & $\begin{array}{l}0.4897 \\
05300\end{array}$ & $\begin{array}{l}0.3418 \\
0.6852\end{array}$ \\
\hline Variances & $\begin{array}{r}2 \\
\sigma_{1 i}^{2} \\
2 \\
\sigma_{2 i}^{2}\end{array}$ & $\begin{array}{l}0.0004 \\
0.0104\end{array}$ & $\begin{array}{l}0.0001 \\
0.0018\end{array}$ & $\begin{array}{l}0.0015 \\
0.0019\end{array}$ & $\begin{array}{l}0.0026 \\
0.0011\end{array}$ & $\begin{array}{l}0.0248 \\
0.2063\end{array}$ & $\begin{array}{l}0.2063 \\
0.0272\end{array}$ & $\begin{array}{l}0.1541 \\
0.1693\end{array}$ & $\begin{array}{l}0.1601 \\
0.0258\end{array}$ \\
\hline $\begin{array}{c}\text { Correlation } \\
\text { Coefficient }\end{array}$ & $\rho_{i}$ & -0.1355 & 0.0338 & -0.0833 & -0.0591 & -0.1669 & 0.0066 & 0.5296 & 0.6843 \\
\hline $\begin{array}{l}\text { Minimum Hue } \\
\text { Minimum Satu }\end{array}$ & & & & $\begin{array}{l}\text { Maxi } \\
\text { Maxi }\end{array}$ & $\mathrm{mH}$ & Valt & $\begin{array}{l}\left(a_{1}\right)= \\
\text { Value }\end{array}$ & $\begin{array}{l}.5152 \\
\left(a_{2}\right)=1\end{array}$ & \\
\hline
\end{tabular}

Table-2.f :Estimated Values of The Parameters For BIRD Image

\begin{tabular}{|c|c|c|c|c|c|c|c|}
\hline \multicolumn{5}{|c|}{ Estimation of Initial Parameters } & \multirow{2}{*}{\multicolumn{3}{|c|}{\begin{tabular}{|c|}
$\begin{array}{c}\text { Estimation of Final Parameters } \\
\text { by E M-Algorithm }\end{array}$ \\
$\begin{array}{c}\text { Number of Image Regions ( } \mathrm{K} \\
=3 \text { ) }\end{array}$
\end{tabular}}} \\
\hline \multirow{2}{*}{\multicolumn{2}{|c|}{ Regions (i) }} & \multicolumn{3}{|c|}{$\begin{array}{l}\text { Number of Image } \\
\text { Regions }(K=\mathbf{3})\end{array}$} & & & \\
\hline & & 1 & 2 & 3 & 1 & 2 & 3 \\
\hline Weights & $\alpha_{i}$ & $1 / 3$ & $1 / 3$ & $1 / 3$ & 0.1029 & 0.6941 & 0.2030 \\
\hline Means & $\begin{array}{l}\mu_{1 i} \\
\mu_{2 i}\end{array}$ & $\begin{array}{l}0.1290 \\
0.6899\end{array}$ & $\begin{array}{l}0.5948 \\
0.1143\end{array}$ & $\begin{array}{l}0.1425 \\
0.2136\end{array}$ & $\begin{array}{l}0.2004 \\
0.7123\end{array}$ & $\begin{array}{l}0.2001 \\
0.4110\end{array}$ & $\begin{array}{l}0.2560 \\
0.3981\end{array}$ \\
\hline Variances & $\begin{array}{r}2 \\
\sigma_{1 i}^{2} \\
\sigma_{2 i}^{2}\end{array}$ & $\begin{array}{l}0.0047 \\
0.0330\end{array}$ & $\begin{array}{c}0.0029 \\
0.0015\end{array}$ & $\begin{array}{c}0.0048 \\
0.0150\end{array}$ & $\begin{array}{l}0.0163 \\
1.9013\end{array}$ & $\begin{array}{l}1.9013 \\
0.2316\end{array}$ & $\begin{array}{l}0.0207 \\
1.7088\end{array}$ \\
\hline $\begin{array}{l}\text { Correlation } \\
\text { Coefficient }\end{array}$ & $\rho_{i}$ & 0.0834 & -0.0504 & -0.1409 & 0.4033 & 0.0460 & 0.5787 \\
\hline $\begin{array}{l}\text { Minimum Hu } \\
\text { Minimum Sa }\end{array}$ & & $\left(\mathbf{b}_{2}\right)=$ & & imur & $\begin{array}{l}\text { Iue Va } \\
\text { Uaturat }\end{array}$ & $\begin{array}{l}\left.i_{1}\right)= \\
\text { alue }\end{array}$ & 1.0000 \\
\hline
\end{tabular}

Substituting the final estimates of the model parameters, the probability density function of the feature vector of each image are estimated.

Using the estimated probability density functions and the image segmentation algorithm given in section 5 , the image segmentation is done for each of the six images under consideration. The original and segmented images are shown in Figure 2.

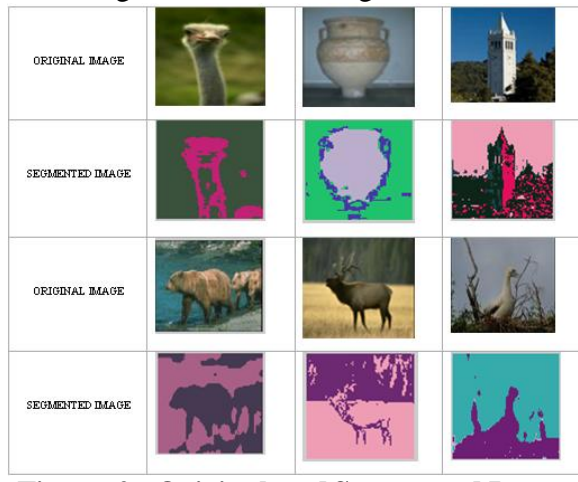

Figure 2: Original and Segmented Images 


\section{PERFORMANCE EVALUATION}

The developed algorithm has been tested on variety of colour images using Berkeley data set images. The performance of the developed algorithm is compared with the segmentation method based on GMM with K-means given by Srinivas Y. et al (2007). The comparison is based on four performance measures namely, Probabilistic Rand Index (PRI) given by Unnikrishnan R. and et al (2007), the Variation of Information (VOI) given by Meila M. (2005), and Global Consistency Error(GCE) given by Martin D. and et al (2001). The objective of the segmentation methods are based on regional similarity measures in relations to their local neighborhood. The qualitative meaning of these performance measures are recalled as follows.

The performance of developed algorithm using finite doubly truncated bivariate Gaussian mixture model (FDTBGMM) is studied by computing the segmentation performance measures namely, PRI, GCE and VOI for the six images under study. The computed values of the performance measures for the developed algorithm and the earlier existing finite Gaussian mixture model (GMM) with K-means algorithm are presented in Table 3 for a comparative study.

Table 3: Segmentation Performance Measures

\begin{tabular}{|c|c|c|c|c|}
\hline \multirow{2}{*}{ IMAGE } & METHOD & \multicolumn{3}{|c|}{ PERFORMANCE MEASURES } \\
\cline { 3 - 5 } & PRI & GCE & VOI \\
\hline \multirow{2}{*}{ POT } & GMM & 0.9234 & 0.4317 & 2.2761 \\
\cline { 2 - 5 } & $\begin{array}{c}\text { FDTBGMM with } K- \\
\text { means }\end{array}$ & 0.9808 & 0.3613 & 0.9461 \\
\cline { 2 - 5 } & $\begin{array}{c}\text { GDMM } \\
\text { mTMM with } K-\end{array}$ & 0.9456 & 0.4281 & 2.5973 \\
\hline \multirow{2}{*}{ TOWER } & GMM & 0.9797 & 0.3984 & 1.7885 \\
\cline { 2 - 5 } & $\begin{array}{c}\text { FDTBGM with } K- \\
\text { means }\end{array}$ & 0.9822 & 0.4226 & 2.6086 \\
\hline \multirow{2}{*}{ B EARS } & GMM & 0.9121 & 0.4418 & 3.2693 \\
\cline { 2 - 5 } & $\begin{array}{c}\text { FDTBGMM with } K- \\
\text { me ans }\end{array}$ & 0.9832 & 0.4330 & 2.6012 \\
\hline \multirow{2}{*}{ DEER } & GMM & 0.9774 & 0.4829 & 2.2863 \\
\cline { 2 - 5 } & $\begin{array}{c}\text { FDTBGMM with } K- \\
\text { means }\end{array}$ & 0.9849 & 0.3990 & 1.2836 \\
\hline \multirow{2}{*}{ B IRD } & GMM & 0.9673 & 0.4671 & 2.7197 \\
\cline { 2 - 5 } & $\begin{array}{c}\text { FDTBGMM with } K- \\
\text { means }\end{array}$ & 0.9719 & 0.4193 & 2.3698 \\
\hline
\end{tabular}

From the above Table 3, it is observed that the developed algorithm is having high PRI and low GCE, VOI compared to finite GMM. This reveals that the proposed algorithm outperforms the existing algorithm based on the Finite Gaussian Mixture Model.

The developed image model and segmentation can also used to reconstruct the image. The Performance Evaluation of the retrieved image is done by Subjective Image Quality testing or by Objective Image Quality testing. The Objective Image Quality testing methods are often used since the numerical results of an objective measure are readily computed and allow a consistence comparison of different algorithms. There are several Image Quality measures available for Performance Evaluation of the Image Segmentation method. An extensive survey of Quality Measures is given by Eskicioglu A.M. and Fisher P.S. (1995). For the Performance Evaluation of the developed Segmentation algorithm, we consider the Image Quality Measures namely (a) Maximum Distance, (b)
Image Fidelity, (c) Mean Square Error, (d) Signal to Noise Ratio and (e) Image Quality Index. Using the estimated probability density functions of the images under consideration the retrieved images are obtained and shown in Figure 3.

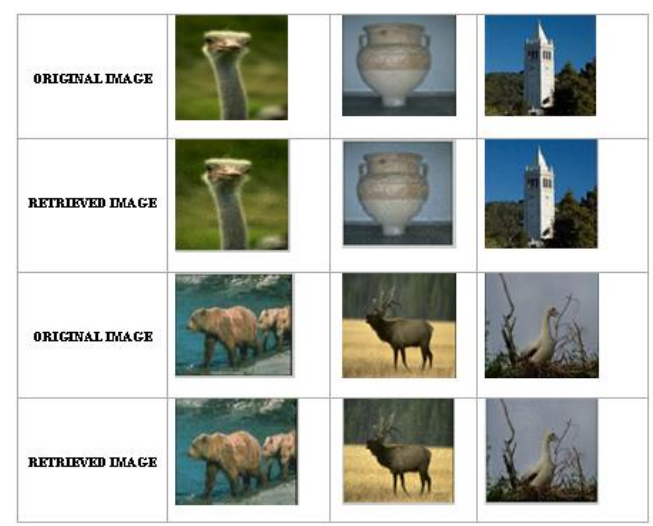

Figure 3: Original and Retrieved Images

The image quality metrics are computed for the six retrieved images OSTRICH, POT, TOWER, BEARS, , DEER and BIRD. Using the proposed model and finite GMM with $K$-means and their values are given in the Table 4.

Table-4: Comparative Study of Image Quality Metrics

\begin{tabular}{|c|c|c|c|c|c|}
\hline IMAGE & Quality Met rics & GMM & \begin{tabular}{|c|} 
FDTB GMM \\
using \\
K-Means
\end{tabular} & $\begin{array}{l}\text { Standard } \\
\text { Limits }\end{array}$ & $\begin{array}{c}\text { Optimal } \\
\text { Criteria }\end{array}$ \\
\hline \multirow{5}{*}{ OSTRICH } & Maxi mu mDistance & 0.5013 & 0.4239 & -1 to +1 & Close to 1 \\
\hline & Image Fidelity & 0.7910 & 0.9088 & 0 to +1 & Close to 1 \\
\hline & Mean Square Error & 0.0932 & 0.0336 & 0 to $\infty$ & Close to 0 \\
\hline & Signal to Noise Ratio & 13.3781 & 15.0687 & 0 to $\infty$ & $\begin{array}{c}\text { As big as } \\
\text { possible }\end{array}$ \\
\hline & Image Quality Index & 0.8102 & 0.8857 & -1 to +1 & Close to 1 \\
\hline \multirow{5}{*}{ POT } & Maxi mu mDistance & 0.3290 & 0.4089 & -1 to +1 & Close to 1 \\
\hline & Image Fidelity & 0.6729 & 0.6762 & 0 to +1 & Close to 1 \\
\hline & Mean Square Error & 0.0738 & 0.0465 & 0 to $\infty$ & Close to 0 \\
\hline & Signal to Noise Ratio & 11.7401 & 12.9939 & 0 to $\infty$ & $\begin{array}{l}\text { As big as } \\
\text { possible }\end{array}$ \\
\hline & Image Quality Index & 0.6075 & 0.6187 & -1 to +1 & Close to 1 \\
\hline \multirow{5}{*}{ TOWER } & Maxi mu mDistance & 0.8481 & 0.9031 & -1 to +1 & Close to 1 \\
\hline & Image Fidelity & 0.5217 & 0.5571 & 0 to +1 & Close to 1 \\
\hline & Mean Square Error & 0.2101 & 0.1227 & 0 to $\infty$ & Close to 0 \\
\hline & Signal to Noise Ratio & 8.8724 & 9.5971 & 0 to $\infty$ & $\begin{array}{c}\text { As big as } \\
\text { possible }\end{array}$ \\
\hline & Image Quality Index & 0.6271 & 0.5965 & -1 to +1 & Close to 1 \\
\hline \multirow{5}{*}{ BEARS } & Maxi mu mDistance & 0.5387 & 0.7991 & -1 to +1 & Close to 1 \\
\hline & Image Fidelity & 0.4277 & 0.6718 & 0 to +1 & Close to 1 \\
\hline & Mean Square Error & 0.0872 & 0.0465 & 0 to $\infty$ & Close to 0 \\
\hline & Signal to Noise Ratio & 9.1217 & 10.8391 & 0 to $\infty$ & $\begin{array}{c}\text { As big as } \\
\text { possible }\end{array}$ \\
\hline & Image Quality Index & 0.5951 & 0.6163 & -1 to +1 & Close to 1 \\
\hline \multirow{5}{*}{ DEER } & Maxi mu mDistance & 0.6217 & 0.6280 & -1 to +1 & Close to 1 \\
\hline & Image Fidelity & 0.3982 & 0.4229 & 0 to +1 & Close to 1 \\
\hline & Mean Square Error & 0.0828 & 0.0564 & 0 to $\infty$ & Close to 0 \\
\hline & Signal to Noise Ratio & 10.0629 & 11.7962 & 0 to $\infty$ & $\begin{array}{c}\text { As big as } \\
\text { possible }\end{array}$ \\
\hline & Image Quality Index & 0.3763 & 0.3684 & -1 to +1 & Close to 1 \\
\hline \multirow{5}{*}{ B IRD } & Maxi mu mDistance & 0.8429 & 0.9347 & -1 to +1 & Close to 1 \\
\hline & Image Fidelity & 0.1920 & 0.1737 & 0 to +1 & Close to 1 \\
\hline & Mean Square Error & 0.2013 & 0.0710 & 0 to $\infty$ & Close to 0 \\
\hline & Signal to Noise Ratio & 8.9231 & 9.0589 & 0 to $\infty$ & $\begin{array}{c}\text { As big as } \\
\text { possible }\end{array}$ \\
\hline & Image Quality Index & 0.3481 & 0.3633 & -1 to +1 & Close to 1 \\
\hline
\end{tabular}


From Table 4, it is observed that all the image quality metrics for the six images are meeting the standard criteria. This implies that using the proposed algorithm the images are retrieved accurately. A comparative study of proposed algorithm with that of algorithm based on Finite Gaussian Mixture Model reveals that the Mean Square Error of the proposed model is less than that of the Finite Gaussian Mixture Model. Based on all other quality metrics also it is observed that the performance of the proposed model in retrieving the images is better than the Finite Gaussian Mixture Model.

\section{CONCLUSION}

In this paper a new image segmentation algorithm is developed and analyzed based on Finite doubly truncated bivariate Gaussian mixture distribution. Here two important characters of the colour image namely, Hue and Saturation are considered as feature vector. Using EM algorithm the parameters are estimated, the K-means algorithm is used to obtain the initial estimates. The segmentation algorithm is developed with component maximum likelihood. The experimentation with Berkeley colour images reveals that this algorithm outperforms the existing algorithms in both image segmentation and image retrievals. The image quality metrics also supported the utility of the proposed algorithm. It is possible to develop image segmentation algorithm with finite mixture of doubly truncated multivariate Gaussian distribution with more image features which require further investigations.

\section{REFERENCES}

[1] Bengt Muthen (1990) "Moments of the censored and truncated bivariate normal distribution", British Journal of Mathematical and Statistical psychology, No.43, pp.131-143.

[2] Cheng et al (2001) "Color Image Segmentation: Advances and Prospects” Pattern Recognition, Vo1.34, pp. 2259-2281.

[3] Dubes R.C. and Jain A.K. (1989), "Random field models in image analysis", Journal of Applied Statistics, Vol.16, No.2.

[4] Eskicioglu A.M. and Fisher P.S. (1995) "Image Quality Measures and their Performance", IEEE Transactions On comm.., Vol.43, No.12, pp.2959-2965.

[5] Feng Zhu et al (2010)," Brain MR image Segmentation based on Gaussian mixture model with spatial information", $3^{\text {rd }}$ International conference on Image and Signal Processing, Vol.3, pp.346-1350.

[6] Jahne (1995), “ A Practical Hand Book on Image segmentation for Scientific Applications, CRC Press.

[7] Juy ong Zhang (2010), "A diffusion approach to seeded image segmentation”, Computer Vision and Pattern Recognition, 2010 IEEE Conference, pp. 2125-2132.

[8] Kokkinos I. and Maragos P. (2009)," Synergy between Object Recognition and Image Segmentation Using the Expectation- Maximization Algorithm", Vol.38, Issue.8, pp. 1486-1501.

[9] Laurent Najman (2011), "On the equivalence between Hierarchical Segmentations and Ultrametric Watersheds", Vol.40, no.3, pp. 231- 247.
[10] Lie T. et al (1993), "Performance evaluation of Finite normal mixture model based image segmentation, IEEE Transactions on Image processing, Vol .12(10), pp.1153-1169.

[11] Martin D., Fowlkes C., Tal D., and Malik J., (2001) “ A database of human segmented natural images and its application to evaluating segmentation algorithms and measuring ecological statistics," in proc. 8th Int. Conference Computer vision, vol.2, pp.416- 423.

[12] Mclanchlan G. and Krishnan T. (1997), "The EM Algorithm and Extensions", John Wiley and Sons, New York -1997.

[13] Mclanchlan G. and Peel D.(2000), “ The EM Algorithm For Parameter Estimations", John Wiley and Sons, New York 2000 .

[14] Meila M. (2005) "Comparing Clustering - An axiomatic view," in proc.22nd Int. Conf. Machine Learning, pp. 577-584.

[15] Modestino J.W. and Zhang J. (1992), "A Markov Random Field Model Based approach to image Interpretation", IEEE Trans. Pattern Annl. Machine Intelligence, Vol.14, No.6, Pg.606-615.

[16] Nor Hazlyna et al (2010), "Comparison of acute Leukemia Image Segmentation using HSI and RGB Color spaces", Information Sciences Signal Processing and their applications, 10th International Conference, pp.749-752.

[17] Norman L. Johnson, Samuel Kortz and Balakrishnan (1994), "Continuous Univariate Distributions" Volume-I, John Wiley and Sons Publications, New York.

[18] Pal S.K. and Pal N.R. (1993), "A Review On Image Segmentation Techniques" , Pattern Recognition, Vol.26, No.9, pp. 1277-1294.

[19] Raut S. et al (2009), "Image Segmentation- A state-of-Art Survey for Prediction ", Advanced Computer control, ICACC '09. International Conference, pp. 420-424.

[20] Rose H.Turi (2001), "Cluster Based Image Segmentation", phd Thesis, Monash University, Australia.

[21] San gwine S.J. and Horne R.E.N. (1998), “The Colour Image Processing Hand Book," Chapmann and Hall, UK.

[22] Srinivas Y. and Srinivas Rao K. (2007), “Unsupervised image segmentation using finite doubly truncated Gaussian mixture model and Hierarchial clustering", Journal of Current Science Vol.93,No.4, pp.507-514.

[23] Srinivas Y. et al (2010)," Unsupervised Image Segmentation Based on Finite Generalized Gaussian Mixture Model With Hierarchical Clustering, International journal for Computational vision and Biomechanics, Vol.3, No.1, pp.73-80.

[24] Tseng, D.C. and Chang C.H. (1992), "Color segmentation usingperceptual attributes," in Proc.11th IEEE International Conference on Pattern Recognition, Vol.3, pp.228-231. 
[25] Udapa J.K et al (1996), "Fuzzy connectedness and object definition: Theory, algorithms, and applications in image segmentation "Graph Models, Image Process, Vol.138, No.3, pp.246-261.

[26] Unnikrishnan R., Pantofaru C., and Hernbert M. (2007), "Toward objective evaluation of image segmentation algorithms," IEEE Trans.Pattern Annl.Mach.Intell, Vol.29 No.6, pp. 929-944.

[27] Un Tang (2010), “A color image segmentation algorithm based on region growing", International conference on Computer Engineering and Technology, Vol.6, pp.V6-634.

[28] Yamazaki T. (1998), "Introduction of EM algorithm into color image segmentation," in Proceedings of ICIRS'98, pp. 368371. International Journal of Signal Processing 6:1
[29] Ye Hou et al (2009), "Image Segmentation Based on GCCV”, $\quad$ HIS' 09. Ninth International conference on Hybrid Intelligent Systems, Vol.1, pp. 252-256.

[30] Zhang Z.H. et al (2003)., “ EM Algorithms for Gaussian Mixtures with Split-and- merge Operation", Pattern Recognition, Vol. 36(9),pp1973-1983.

[31] Zhenh a Zhang et al (2009), “An improving Technique of Color Histogram in Segmentation-based Image Retrieval "IAS '09. $5^{\text {th }}$ International Conference, Vol.2, pp. 381-384.

[32] Zhen Wang and Meng Yang (2010), "A fast Clustering algorithm in Image Segmentation", 2nd International conference on Computer Engineering and Technology, Vol.6, pp. V6-592- V6-594. 